\title{
Multicomponent Lattice Boltzmann Simulations of Gas Transport in a Coal Reservoir with Dynamic Adsorption
}

\author{
Zhigao Peng $\mathbb{D}$, ${ }^{1}$ Shenggui Liu $\mathbb{D}^{1},{ }^{1}$ Songlei Tang $\mathbb{D},{ }^{1}$ Yuechao Zhao $\mathbb{D}{ }^{2}$, and Yingjun Li $^{3}{ }^{3}$ \\ ${ }^{1}$ School of Mechanics \& Civil Engineering, China University of Mining and Technology (Beijing), Beijing 100083, China \\ ${ }^{2}$ College of Resources \& Safety Engineering, China University of Mining and Technology (Beijing), Beijing 100083, China \\ ${ }^{3}$ State Key Laboratory for Geomechanics and Deep Underground Engineering, China University of Mining and Technology (Beijing), \\ Beijing 100083, China
}

Correspondence should be addressed to Shenggui Liu; liushg2002@163.com and Yingjun Li; lyj@aphy.iphy.ac.cn

Received 1 March 2018; Revised 15 May 2018; Accepted 29 May 2018; Published 12 July 2018

Academic Editor: Mandadige S. A. Perera

Copyright (C) 2018 Zhigao Peng et al. This is an open access article distributed under the Creative Commons Attribution License, which permits unrestricted use, distribution, and reproduction in any medium, provided the original work is properly cited.

\begin{abstract}
Gas adsorption occurs when the dynamic adsorption equilibrium conditions of the local adsorptive sites are broken. In the overall process of unconventional natural gas generation, enrichment, storage, and production, this phenomenon plays a significant role. A double-distribution Lattice Boltzmann model for solving the coupled generalized Navier-Stokes equation and advection-diffusion equation with respect to the gas-solid dynamic adsorption process is proposed for multicomponent gas migration in the unconventional reservoir. The effective diffusion coefficient is introduced to the model of gas transport in the porous media. The Langmuir adsorption rate equation is employed to control the adsorption kinetic process of gas-solid adsorption/desorption. The model is validated in two steps through fluid flow without and with gas diffusion-adsorption between two parallel plates filled with porous media, respectively. Simulation results indicate that with other parameters being equal, the rate of gas diffusion in the porous material and the area of the dynamic adsorption equilibrium-associated region increase with the matrix porosity/permeability. Similar results will happen with a greater saturation adsorption amount or a lower Langmuir pressure. The geometric effect on adsorption is also studied, and it is found that a higher specific surface area or free flow region can enhance the gas transport and the rate of adsorption.
\end{abstract}

\section{Introduction}

Coalbed methane (CBM) is one of the most important and potential energies which has been recognized as an alternative to oil and has achieved more and more attention in recent years [1]. Different with the conventional natural gas, a coal seam is both a resource and reservoir of CBM and stores methane in nanopores in the form of adsorption, resulting in the low recovery of CBM exploitation [2]. Nowadays, understanding the mechanism of gas storage and transport in the reservoir is of vital importance for current research and practical application.

Coal is one of the most complex solids, and its physical property varies from the depth, hydrogeology, reservoirforming process, and so on, which leads to a highly heterogeneous structure with pore size ranging from a few nanometers to over a micrometer. According to the classification scheme proposed by the International Union of Applied Chemistry (IUPAC) [3] and Hodot [4], pores are divided into three categories based on size: micropores (less than $2 \mathrm{~nm}$ ), mesopores (from 2 to $50 \mathrm{~nm}$ ), and macropores (from 50 to $10^{4} \mathrm{~nm}$ ), as well as microfractures (more than $10^{4} \mathrm{~nm}$ ). Under the existing research [2], the coal contains massive micropores, some mesopores, and little macropores. Micropores and mesopores provide space for storing most of the adsorbed molecules. Macropores are of no significance in terms of adsorption capacity in that the area contribution is very small and can usually be negligible compared to the area contributed by the micropores/mesopores; they always act as transport pores to allow adsorbate molecules to diffuse from the bulk phase into the particle interior [5].

Generally, coal is characterized as a dual-pore system [6] including the matrix porosity consisting of micropores/ mesopores in the coal matrix (i.e., the porous material 
region) and the fracture porosity composed of nonuniformly distributed macropores and microfractures (i.e., the free flow region) [7]. The adsorption processes of methane in the reservoir can be divided into two parts: Enter the irregular macropore- microfractures system by advection-diffusion; diffuse in the nanopore network of a matrix, then adsorbed on the interior surface of pores. The heterogeneity of geometric shapes, the difference of pore size/distribution, and the involved multiple transport mechanism of fluid causing the difficulty of the research and causing the fluid flow and transport in the reservoir are usually treated at the representative elementary volume (REV) scale in experiments and numerical simulation [8-12]. Recently, the Lattice Boltzmann method (LBM) has been widely used in the field relevant to fluid flow and gas transport at the mesoscale because of its efficiency and effectiveness in the implementation of multiple interparticle interactions and complex geometry boundary conditions [13-15]. Nithiarasu et al. [16] proposed a generalized Navier-Stokes equation for isothermal incompressible flow in porous media, which ignores the detailed structure of the porous media by including an additional term to account for the presence of a porous medium. Guo and Zhao [9] developed an LB model which can be used to solve the generalized Navier-Stokes equations. Then, Chen et al. [8] employed the generalized Lattice Boltzmann (GLB) model with the Klinkenberg effect in shale gas for studying the gas slippage and its effect on apparent permeability. Ning et al. [12] and Wang et al. [11] used a similar model for studying the impact of surface adsorbed gas on apparent permeability based on $2 \mathrm{D}$ and $3 \mathrm{D}$ reconstructed shale, respectively. Liu and $\mathrm{He}$ [17] developed a nonorthogonal multirelaxation time (MRT) LB model with the GLB equation for investigating convection heat transfer in porous media.

As for the coal gas adsorption process, in general, it is considered as a physisorption process because the molecular forces involved are normally of the Van der Waals type [2, 7]. Many studies, including both experiment and numerical simulation, have focused on (isothermal) adsorption equilibrium without concerning the intermediate states or time in recent years $[11,18]$. However, the isothermal adsorption equilibrium can only provide static information about the adsorption process at a specific stage, which is insufficient to reflect the kinetic behavior of the coal gas stored in the reservoir. In adsorption kinetics, the concentration changes associated with adsorption were related to the time variable. Therefore, it is for the benefit of studying the effect of various factors, such as the gas content, capacity, porosity, and permeability, on the adsorption process. Recently, more and more researchers concentrated on developing an LB model for multiple transport mechanisms with chemical reaction in the porous media. He et al. [19] proposed an LB model for fluid diffusion-convection with a chemical kinetic reaction using the double distribution function for controlling the fluid flow and diffusion, which introduced a source/sink term in the diffusion equation to govern the reaction process. Tian et al. [10] proposed a coupled LB model using the double distribution function with the GLB equation to describe the geochemical reactions during $\mathrm{CO}_{2}$ injection. It is essential that a novel LB model be developed not only for multiple transport mechanism investigation on methane migration in the reservoir but also for practical reservoir upscaling techniques used in unconventional natural gas exploitation.

In the present work, we developed a double-distribution Lattice Boltzmann model to solve a coupled generalized Navier-Stokes equation, and the advection-diffusion equation is proposed for unconventional natural gas migration in the reservoir that includes gas-solid adsorption and desorption, which is based on the REV model of Guo and Zhao [9] and the diffusion-reaction model of He et al. [19]. The model mainly contains three parts: (1) the fluid flow at the REV scale which is governed by the generalized NavierStokes equations, (2) the mass transfer in porous media with respect to the effective diffusivity and a source/sink term solved by the advection-diffusion equation, and (3) the gassolid adsorption process in the porous matrix which is governed by the typical Langmuir adsorption rate equation. The remaining parts are organized as follows: In Section 2, a generalized LB model for fluid flow in porous media is revisited briefly. A passive scalar LB equation with adsorption is presented in Section 3. Numerical results and discussion are given in Section 4. Finally, a brief conclusion is made in Section 5.

\section{Generalized Model for Fluid Flow in Porous Media}

For isothermal flows of incompressible fluids in porous media at the REV scale, the generalized Navier-Stokes equation which was proposed by Nithiarasu et al. is capable for the simulation.

2.1. Generalized Navier-Stokes Equation. The governing equations of mass and momentum for the generalized Navier-Stokes equations can be given by

$$
\begin{aligned}
\nabla \cdot \mathrm{u} & =0 \\
\frac{\partial \mathrm{u}}{\partial t}+(\mathbf{u} \cdot \nabla)\left(\frac{\mathbf{u}}{\varepsilon}\right) & =-\frac{1}{\rho} \nabla(\varepsilon p)+v_{\mathrm{e}} \nabla^{2} \mathbf{u}+\mathrm{F}
\end{aligned}
$$

where $\mathrm{u}$ is the fluid velocity, in $\mathrm{m} / \mathrm{s} ; \varepsilon$ is the porosity; $\rho$ is the fluid density, in $\mathrm{kg} / \mathrm{m}^{3} ; p$ is the pressure, in $\mathrm{Pa} ; v_{\mathrm{e}}$ is the effective kinematic viscosity, in $\mathrm{m}^{2} / \mathrm{s}$, where $\nu_{\mathrm{e}}=v J$ and $J$ is the viscosity ratio; and $\mathrm{F}$ represents the total body force including both medium resistance and external forces and can be given by

$$
\mathrm{F}=-\frac{\varepsilon v}{K} \mathrm{u}-\frac{\varepsilon F_{\varepsilon}}{\sqrt{K}}|\mathrm{u}| \mathrm{u}+\varepsilon \mathrm{G}
$$

where $\mathrm{G}$ is the external body force, in $\mathrm{N} ; F_{\varepsilon}$ is the geometric function, in $\mathrm{N}$; and $K$ is the permeability of porous media, in $\mathrm{m}^{2}$.

Both $F_{\varepsilon}$ and $K$ are related to the porosity. For a porous medium composed of solid particles, the Ergun correlation gives (Ergun 1952) 


$$
\begin{aligned}
& F_{\varepsilon}=\frac{1.75}{\sqrt{150 \varepsilon^{3}}}, \\
& K=\frac{\varepsilon^{3} d_{\mathrm{p}}^{2}}{150(1-\varepsilon)^{2}},
\end{aligned}
$$

where $d_{\mathrm{p}}$ is the diameter of the solid particle, in $\mathrm{m}$.

2.2. LB Model for Generalized Navier-Stokes Equations. Guo and Zhao [9] constructed an LBE model which can be used to solve the generalized Navier-Stokes equations; the corresponding evolution equation of the particle distribution function is

$$
\begin{aligned}
f_{i}\left(x+\mathbf{e}_{i} \delta_{\mathrm{t}}, t+\delta_{\mathrm{t}}\right)-f_{i}(x, t)=-\frac{1}{\tau}\left[f_{i}(x, t)-f_{i}^{\mathrm{eq}}(x, t)\right]+\delta_{t} \mathbf{F}_{i}, \\
f_{i}^{\mathrm{eq}}=\rho w_{i}\left[1+\frac{\mathbf{e}_{i} \cdot \mathbf{u}}{c_{s}^{2}}+\frac{\mathbf{u} \mathbf{u}:\left(\mathbf{e}_{i} \mathbf{e}_{i}-c_{s}^{2} \mathbf{I}\right)}{2 \varepsilon c_{s}^{4}}\right] \\
\mathbf{F}_{i}=\rho w_{i}\left(1-\frac{1}{2 \tau}\right)\left[\frac{\mathrm{e}_{i} \cdot \mathrm{F}}{c_{s}^{2}}+\frac{\mathrm{uF}:\left(\mathrm{e}_{i} \mathrm{e}_{i}-c_{s}^{2} \mathrm{I}\right)}{\varepsilon c_{s}^{4}}\right]
\end{aligned}
$$

where $f_{i}$ is the discrete density distribution function, $f_{i}^{\text {eq }}$ is the local equilibrium equation, $\mathrm{e}_{i}$ is the discrete velocity of particles, $\delta_{\mathrm{t}}$ is the time step, $\tau$ is the relaxation time, $\mathrm{I}$ is the unit matrix, $c_{s}$ is the sound velocity of the lattice, and $w_{i}$ is the weighting coefficient.

As in the standard LBE, the density and velocity of flow are defined as

$$
\begin{aligned}
\rho & =\sum_{i} f_{i}, \\
\rho \mathbf{u} & =\sum_{i} f_{i} \mathbf{e}_{i}+\frac{\delta_{\mathrm{t}}}{2} \rho \mathbf{F} .
\end{aligned}
$$

Because the force $\mathrm{F}$ also contains the flow velocity, the velocity u can be explicitly given by

$$
\mathrm{u}=\frac{\mathrm{v}}{c_{0}+\sqrt{c_{0}^{2}+c_{1}|\mathrm{v}|}}
$$

where $\mathbf{v}$ is a temporary velocity defined by

$$
\rho \mathbf{v}=\sum_{i} f_{i} \mathbf{e}_{i}+\frac{\delta_{\mathrm{t}}}{2} \varepsilon \rho \mathbf{G},
$$

and the parameters $c_{0}$ and $c_{1}$ are given by

$$
\begin{aligned}
& c_{0}=\frac{1}{2}\left(1+\varepsilon \frac{\delta_{\mathrm{t}}}{2} \frac{v}{K}\right), \\
& c_{1}=\varepsilon \frac{\delta_{\mathrm{t}}}{2} \frac{F_{\varepsilon}}{\sqrt{K}} .
\end{aligned}
$$

By using the Chapman-Enskog technique with the pressure $p=c_{s}^{2} \rho / \varepsilon$ and the effective viscosity $v_{\mathrm{e}}=c_{s}^{2}(\tau-0.5) \delta_{\mathrm{t}}$, the generalized LB model can recover to (1) and (2) in the incompressible limit.

\section{Governing Equation for Gas Transport with Adsorption/Desorption}

3.1. The Advection-Diffusion Equation for Mass Transfer. Generally, gas transport in porous media can be considered as a mass transfer process [20-22], which can be expressed by the advection-diffusion equation

$$
\frac{\partial C}{\partial_{\mathrm{t}}}+\nabla \cdot(C \mathbf{u})=\nabla \cdot\left(D_{s} \nabla C\right)+R_{\mathrm{s}},
$$

where $C$ is the concentration of the gas phase, in $\mathrm{mol} / \mathrm{m}^{3} ; D_{s}$ is the diffusion coefficient, in $\mathrm{m}^{2} / \mathrm{s} ; R_{\mathrm{s}}$ is the resource/sink term, which is involved in the adsorption-desorption process, in $\mathrm{mol} /\left(\mathrm{m}^{3} \cdot \mathrm{s}\right)$.

The diffusion coefficient $D_{s}$ is a key variable describing the gas transport capacity in porous media and is determined by the physical property of the porous material. Since the pore size included in the organic matrix is in the order of nanometers, the diffusion process which is generally called Knudsen diffusion is quite complicated compared to the gas diffusion in the free flow region and should be considered separately. By considering diffusive scattering for gas transport in a simple geometric capillary with a length $L$ and radius $r$, Kang et al. [24] estimated the Knudsen diffusion coefficient

$$
D_{K}=\frac{2 r}{3} \sqrt{\frac{8 R T}{\pi M}},
$$

where $r$ is the mean pore radius of the coal matrix, in $\mathrm{m}$; $R$ is the gas constant, in $\mathrm{J} /(\mathrm{mol} \cdot \mathrm{K})$; $T$ is the temperature, in $\mathrm{K}$; and $M$ is the gas molar mass, in $\mathrm{kg} / \mathrm{mol}$.

However, the measurement of diffusivity is the outcome of an expression derived from a single capillary pore, which will be somewhat larger than the experimentally obtained diffusion coefficient. Typically, for gas transport in porous media, the porosity/tortuosity ratio should be related to diffusivity. Therefore, we can roughly obtain the effective diffusion coefficient [24]

$$
D_{\text {eff }}=\left(\frac{\phi_{k}}{\phi_{\text {Toc }}}\right)\left(\frac{\varepsilon}{\sigma^{2}}\right) D_{K},
$$

where $\phi_{k} / \phi_{T o c}$ is the organic/total volume ratio and $\sigma$ is the tortuosity.

3.2. The Rate Equation for Adsorption-Desorption Kinetics. Gas migration through solid media is always accompanied with gas-solid adsorption-desorption due to the local concentration fluctuant breaking down the adsorption equilibrium. In order to reveal the gas transport behavior with adsorption in a coal gas reservoir, this paper employed the typical Langmuir adsorption rate equation which is widely used in unconventional natural gas industries and is applicable for gas adsorption at the nanoscale $[19,25,26]$ :

$$
\frac{\partial V}{\partial_{\mathrm{t}}}=k_{\mathrm{a}} C\left(V_{m}-V\right)-k_{\mathrm{d}} V,
$$


where $k_{\mathrm{a}}$ is the adsorption rate constant, in $\mathrm{m}^{3} /(\mathrm{mol} \cdot \mathrm{s}) ; k_{\mathrm{d}}$ is the desorption rate constant, $1 / \mathrm{s} ; V$ is the absorbed amount, in $\mathrm{mol} / \mathrm{m}^{3}$; and $V_{m}$ is the saturated adsorption capacity, in $\mathrm{mol} / \mathrm{m}^{3}$.

Adsorption equilibrium information is the most important part of the adsorption process, when $\partial V / \partial_{t}=0$; (13) can be recovered as the equivalent Langmuir isothermal adsorption equilibrium equation

$$
V=\frac{V_{m} p_{c}}{p_{c}+p_{\mathrm{L}}}
$$

where $p_{c}$ is the pressure of the field, where $p_{c}=C M c_{s}^{2}$, in $\mathrm{Pa}$; $P_{\mathrm{L}}$ represents the Langmuir pressure, where $p_{\mathrm{L}}=c_{s}^{2} M k_{\mathrm{d}} / k_{\mathrm{a}}$, in $\mathrm{Pa}$.

In the Langmuir kinetic equation, the magnitude of $k_{\mathrm{a}}$ and $k_{\mathrm{d}}$ fixes the adsorption/desorption rate; meanwhile, $k_{\mathrm{d}} / k_{\mathrm{a}}$ determines the slope of the isothermal adsorption curve, thus any two of them can determine the third. It is obvious that the rate of adsorption $R_{\mathrm{a}}=k_{\mathrm{a}} C\left(V_{m}-V\right)$ is a concentration-dependent parameter and the rate of desorption $R_{\mathrm{d}}=k_{\mathrm{d}} V$ is in proportion to the adsorbed amount.

In order to incorporate the gas-solid adsorption, the approach of $\mathrm{He}$ et al. [19] was modified to include the selfadaptive conversion between free gas and adsorbed gas. Under this situation, each lattice node corresponding to the porous media is considered a well-adsorptive site. Therefore, the Langmuir rate equation can be integrated into the source/ sink term in (10) as follows:

$$
R_{\mathrm{s}}=\frac{\partial V}{\partial_{\mathrm{t}}}=k_{\mathrm{a}} C\left(V_{m}-V\right)-k_{\mathrm{d}} V
$$

In the evolution process, the adsorbed amount was updated over time; the new amount can be obtained using the first-order difference scheme

$$
V_{\mathrm{t}+1}-V=\delta_{\mathrm{t}}\left[k_{\mathrm{a}} C\left(V_{m}-V\right)-k_{\mathrm{d}} V\right]
$$

It must be mentioned that a broad variety of pore size and distribution exists in the coal matrix, which increases the anisotropic and complexity of fluid flow, gas diffusion, and gas-solid physisorption. However, for upscaling and computational efficiency purposes, we can simplify the porous media with homogenization theory due to the material of porous media, the pressure, and the concentration which will just fluctuate in a few ranges at the REV scale.

\subsection{LB Model for Gas Transfer with Adsorption in Porous} Media. For 2D simulation of the species transfer process, by ignoring velocities at the $\mathrm{D} 2 \mathrm{Q} 9$ diagonals, the model can be reduced to the D2Q5 LB model and will not lose accuracy; thus, the following D2Q5 LB equation is employed to control mass transfer:

$$
g_{i}\left(x+\mathbf{e}_{i} \delta_{\mathrm{t}}, t+\delta_{\mathrm{t}}\right)-g_{i}(x, t)=-\frac{1}{\tau_{g}}\left[g_{i}(x, t)-g_{i}^{\mathrm{eq}}(x, t)\right]+\omega_{i} R_{\mathrm{s}} \delta_{\mathrm{t}},
$$

$$
g_{i}^{\mathrm{eq}}=C w_{i}\left[1+\frac{\mathbf{e}_{i} \cdot \mathbf{u}}{c_{s}^{2}}\right]
$$

where $g_{i}$ is the discrete density distribution function of mass transfer, $g_{i}^{\mathrm{eq}}$ is the corresponding local equilibrium equation, and $\tau_{g}$ is the relaxation time of mass transfer.

Similarly, the concentration of (18) is given by

$$
C=\sum_{i} g_{i}
$$

and with $D_{s}=c_{s}^{2}\left(t_{g}-0.5\right) \delta_{\mathrm{t}}$, (17) can be recovered as (10).

\section{Results and Discussion}

Before the investigation, a schematic diagram is displayed in Figure 1 to explain the adsorption process of coalbed methane in the reservoir. Generally, gas stored in the coal matrix mainly contains three forms: free gas, adsorbed gas, and dissolved gas.

The gas molecules of coalbed methane migrate with the fluid in the free flow region, a part of the free gas molecules will be trapped by the solid wall when they reached the interface of the free flow region and the porous medium, and more molecules enter the nanopore network and are adsorbed on the inner surface of pores. In this process, both gas adsorption and desorption exist due to the fluctuant of the local concentration, which leads to the exchange of gas molecules between pores and the wall. The detailed information of coalbed methane storage in the reservoir can be seen in Figure 1.

4.1. Flow between Two Parallel Plates Filled with a Porous Media. To validate the present LB model, we simulate the fluid flow between two parallel plates filled with a porous medium which is a homogeneous material with a uniform porosity $\varepsilon$ and permeability $K$. In the simulation, the computational grid is $100 \times 100$, the relaxation time $\tau$ is 0.8 , and the viscosity ratio $J$ is assumed to be unity. The flow is driven by a pressure difference, and a nonslip boundary condition is applied to both the top and bottom walls.

Firstly, we suppose there was only one single component fluid flow in the media, then the nonlinear resistance forces [the second term in Eq. (3)] can be ignored due to the fluid flow is weak and the inertial is disappeared. Consequently, the flow at steady state can be described by the Brinkmanextended Darcy equation

$$
\frac{v_{\mathrm{e}}}{\varepsilon} \frac{\partial^{2} u}{\partial y^{2}}-\frac{v}{K} u+G=0
$$

with $u(x, 0)=0$ and $u(x, H)=0$; the analytical solution can be given by

$$
u=\frac{G K}{v}\left(1-\frac{\cosh [r(y-H / 2)]}{\cosh (r H / 2)}\right), \quad r=\sqrt{v \varepsilon / K v_{\mathrm{e}}}
$$

where $H$ is the width of the field, and cosh is the hyperbolic function with $\cosh (x)=e^{x}+e^{-x} / 2$. 


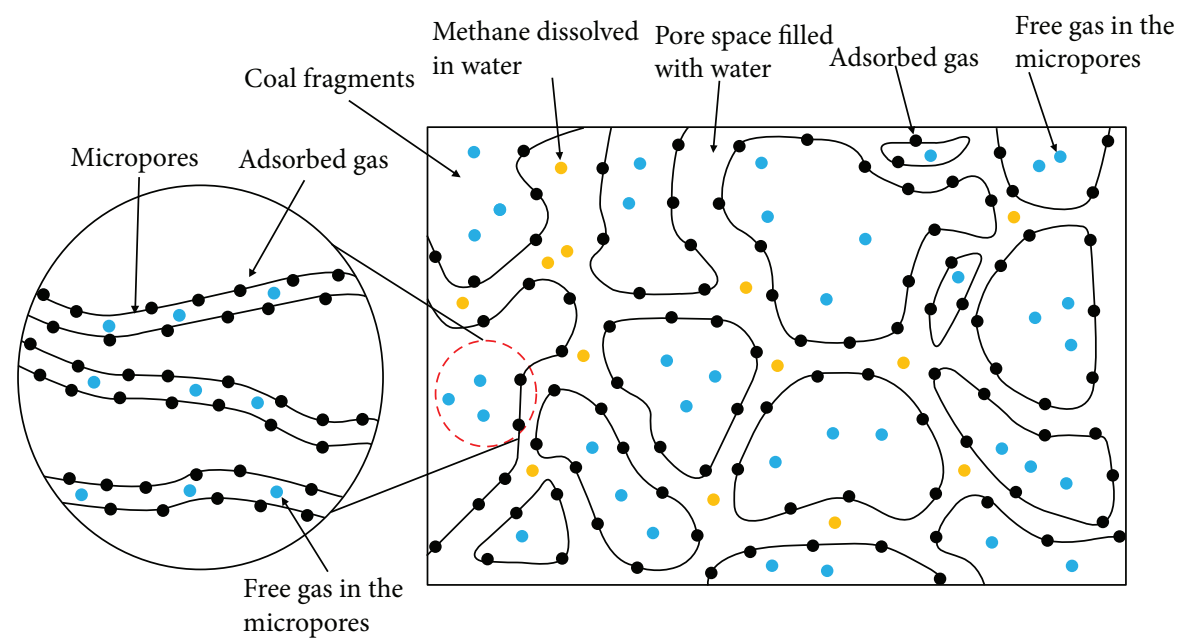

FIGURE 1: Diagram of coalbed methane storage in the reservoir.

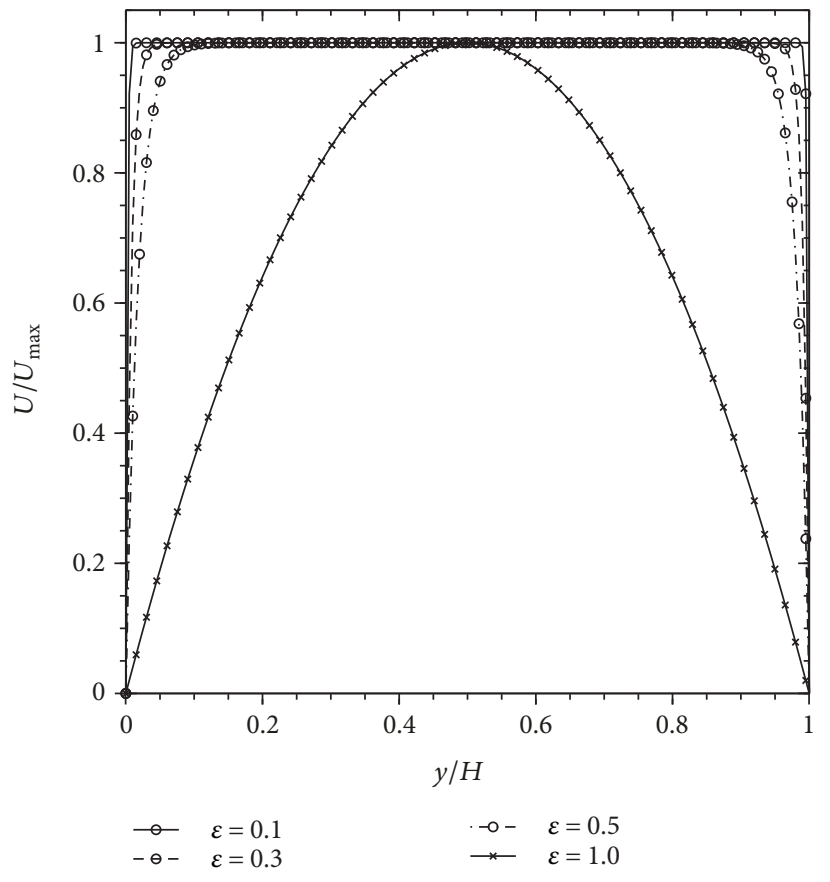

(a)

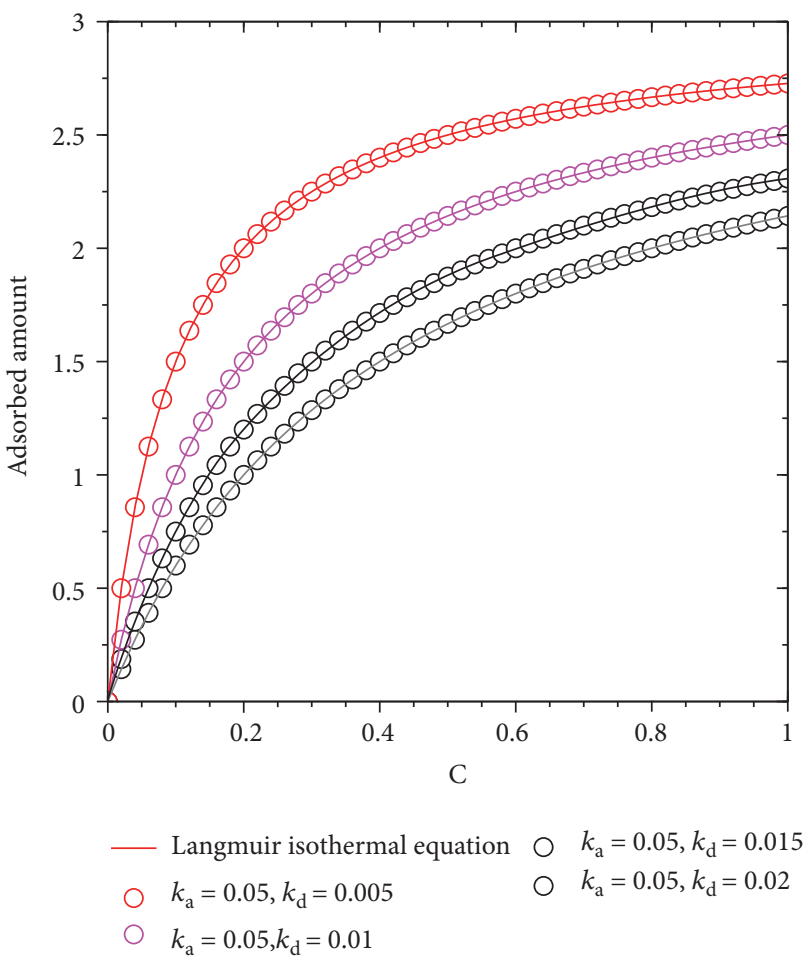

(b)

FigURE 2: Simulation results of porous flow between plates. (a) The velocity profiles of a single-component fluid flow without mass transfer, ignoring the nonlinear drag force term. Note that velocity $u$ was normalized by the maximum velocity at the centerline of the channel $u_{\max }$. (b) The relationship between the concentration and the adsorbed amount of coupled flow with both fluid flow and gas diffusion. The results (symbols) are in good agreement with the analytical solution (lines).

The velocity profiles are detailed in Figure 2(a). When the porosity is 1.0, the fluid flow between the two plates is the free flow due to the porous material being absent. The results are consistent with the analytical solution of the typical plane Poiseuille flow. Owing to the existence of porous media, with the reduction in porosity, the velocity profiles will be more flattened, which is in good agreement with the solution of the Brinkman-extended Darcy equation.
Then, the diffusion field with adsorption effect is added for the validation of the coupling system. In this case, the diffusion coefficient is $1 / 3$, and a constant concentration $C_{0}=1$ is set at the inlet; the initial concentration of the rest of the field is 0 , the saturation adsorption capacity is 3 , the desorption rate constants are set to $0.005,0.01,0.015$, and 0.02 , and the adsorption rate constant is fixed at 0.05. It is worthwhile mentioning that the nonlinear resistance forces should be 
included due to the system involving mass transfer through the fluid-solid interface.

Figure 2(b) displays the relationship between the concentration and the adsorbed amount of the coupled flow with both fluid flow and gas diffusion. At a fixed value of concentration, the higher the Langmuir pressure, the lower the adsorbed amount, which can be supported by the solution of a typical Langmuir adsorption isothermal equation.

It is obvious that all the simulation results are in good agreement with the analytical solution in Figure 2, which ensures the validation of the present model.

\subsection{Geometric Effects of Coal Matrix on the Adsorption} Process. The pore structure of a real solid of the coal reservoir is so complex that we have to model (idealize) the structure so that it can be represented mathematically, which makes the mathematics of diffusion and adsorption tractable. On the other side, it is essential that the porous media should be reasonably complex enough to bring out the features of the solid and their influence on diffusion and adsorption. For a convenient implementation of the numerical investigation, the coal matrix is usually characterized by porous media in two ways: a matrix with regularly distributed square solid blocks and a pseudorealistic field which is randomly divided by several irregular cleats. The first one is simple enough for ignoring the influence of geometry, and the second one is a general method for simulating fluid flow through a heterogeneous porous media. Actually, the coal seam contains both organic and inorganic matters, the gassolid adsorption always takes place in the organic parts, and the inorganic media only act as the microchannel for fluid flow. This paper ignores the influence of the inorganic parts due to the computational domain being at a relatively small scale.

In reality, the output concentration is the parameter we always focus on in practical applications and in engineering; meanwhile, the methane-coal adsorption is a concentrationdependent process owing to the fact that the intrinsic adsorption rate is usually much faster than the diffusion rate [5]. Therefore, it is acceptable to evaluate the effect of geometric and Langmuir parameters on adsorption through the change in concentration distribution. In this section, the porous structures G-1 $\left(S=0.4, \varepsilon_{f}=0.64\right)$, G-2 $\left(S=0.27, \varepsilon_{f}=0.64\right)$, G-3 $\left(S=0.13, \varepsilon_{f}=0.64\right), \mathrm{G}-4\left(S=0.13, \varepsilon_{f}=0.55\right)$, and G-5 $\left(S=0.13, \varepsilon_{f}=0.44\right)$ were used to study the effect of porosity of microfractures and the specific surface area of the organic matrix on dynamic diffusion-adsorption processes. In order to maintain the connectivity of the $2 \mathrm{D}$ reconstructed structure and achieve an economic computation time without losing physical interpretation, a relatively high fracture porosity is assumed [27].

Note that there are two kinds of porosity in our simulation: the matrix porosity $\varepsilon$ and the fracture porosity $\varepsilon_{f}$, the former one is a static averaged data which reflects the level of nanopores of the organic matrix, and another is the ratio between the area of the free flow region (microfractures) and the total area, which represents the magnitude of the area of microfractures and ignores the void within the porous
TABLE 1: Input parameters in simulation.

\begin{tabular}{lc}
\hline Simulation domain $(u m \times u m)$ & $16 \times 16$ \\
\hline Temperature $T(\mathrm{~K})$ & 303.15 \\
Reservoir pressure $P_{0}(\mathrm{MPa})$ & 10 \\
Pressure gradient $(\mathrm{MPa} / \mathrm{m})$ & 0.1 \\
The viscosity of methane $(\mathrm{Pa} \cdot \mathrm{s})$ & $1.39 \times 10^{-6}$ \\
Concentration at inlet $C_{0}\left(\mathrm{~mol} / \mathrm{m}^{3}\right)$ & 0.1 \\
Matrix porosity $\varepsilon$ & $0.05 \sim 0.4$ \\
The diffusion coefficient of free flow region $D_{\mathrm{sf}}\left(\mathrm{m}^{2} / \mathrm{s}\right)$ & $1.59 \times 10^{-5}$ \\
Knudsen diffusion coefficient $D_{K}\left(\mathrm{~m}^{2} / \mathrm{s}\right)$ & $1.69 \times 10^{-6}$ \\
$\varnothing_{k} / \varnothing_{T o c} / \sigma^{2}$ & 0.0016 \\
Saturation adsorption amount $V_{m}\left(\mathrm{~m}^{3} / \mathrm{t}\right)$ & $10 \sim 30$ \\
Adsorption rate constant $k_{\mathrm{a}}\left(\mathrm{m}^{3} /\left(\mathrm{mol}^{2} \cdot \mathrm{s}\right)\right)$ & $1.0 \times 10^{3}$ \\
Desorption rate constant $k_{\mathrm{d}}(/ \mathrm{s})$ & $3.78 \times 10^{7}$ \\
Langmuir pressure $p_{\mathrm{L}}(\mathrm{MPa})$ & $1 \sim 4$ \\
\hline
\end{tabular}

Note that relevant parameters were selected from typical geologic exploration data of the Qinshui Basin in China [28]. The adsorption and desorption rate constants were referred from the literature $[29,30]$.

material. The specific surface area $S$ is the ratio between the length and area of the porous solid.

In the simulation, the domain is discretized by $200 \times 200$ lattices, with a resolution of $80 \mathrm{~nm}$; the reference temperature for adsorption capacity is chosen as $303.15 \mathrm{~K}$. For the fluid flow process, the no-slip boundary condition is used for the top and bottom walls and the interface between the free flow region and the solid matrix, and a pressure difference is set between the inlet and the outlet. Two kinds of boundary conditions are employed for methane transport: constant concentration $\left(C=C_{0}\right)$ at the inlet and Neumann boundary condition $(\partial C / \partial \mathrm{n}=0)$ for the other three boundaries. The initial concentration in the domain is 0 . Other input parameters are detailed in Table 1. It must be mentioned that the effect of matrix porosity (porosity of the porous material), particle size, and distribution inside the porous media is ignored due to the statistical averaged parameters used, which can be ascribed to the reservoir upscaling technique included in our model.

Figure 3 shows the concentration distribution profile with different fracture porosities and specific surface areas, respectively. The structures of G1 to G3 have different specific surface areas but the same porosities. With an increased specific surface area, the concentration difference between the free flow region and the porous material is smoother, which means gas fully entered in the coal matrix and reached the dynamic adsorption equilibrium consuming less time, due to the small size and wide distribution of the porous blocks. The structures of G3 to G5 have different fracture porosities but the same specific surface areas. With increased porosity, the difference in concentration magnitude distribution inside porous blocks of each structure is not significant; however, the rate of gas transfer through the entire domain was more efficient, due to the higher fracture porosity representing a more extensive free flow region, which leads to the lowering of both the adsorbed amount per time and the total 


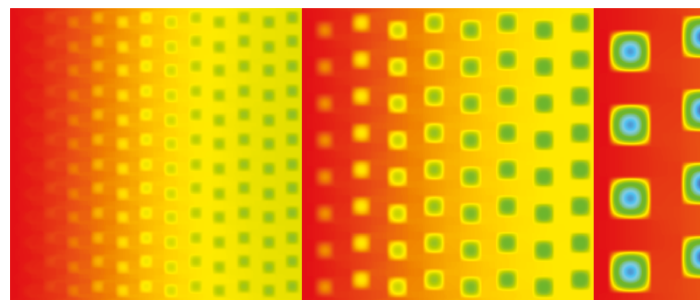

(a) (b)

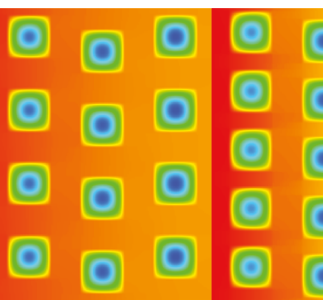

(c)

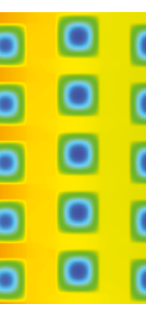

(d)

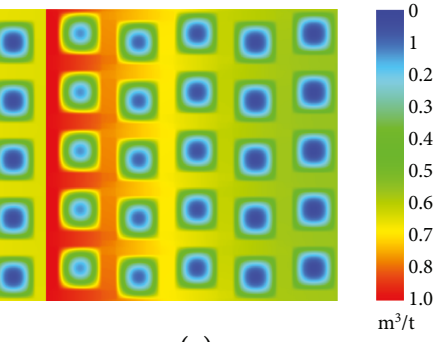

(e)

FIGURE 3: Results of the concentration distribution profile for methane migration in porous media with adsorption under different porosities and specific surface areas, respectively. (a) G-1 $\left(S=0.4, \varepsilon_{f}=0.64\right)$, (b) G-2 $\left(S=0.27, \varepsilon_{f}=0.64\right)$, (c) G-3 $\left(S=0.13, \varepsilon_{f}=0.64\right),(\mathrm{d}) \mathrm{G}-4(S=0.13$, $\left.\varepsilon_{f}=0.55\right)$, (e) G-5 $\left(S=0.13, \varepsilon_{f}=0.44\right)$. The matrix porosity of the solid matrix is 0.1 .

adsorbed amount of porous solid. Besides, the decreased total area of porous media may result in a relatively smaller medium resistance to gas transport. These phenomena can also be confirmed in Figure 4.

A larger specific surface area $S$ means that the uniformly distributed porous solid will lead to higher medium resistance; thus, the rate of output concentration is maintained at a lower level as can be seen in Figure 4 and then increased due to the widely distributed small size of porous blocks, making the adsorption reach the equilibrium state at a shorter time. However, as mentioned above, a larger fracture porosity represents the higher area of microfractures, leading to the output concentration increasing with $\varepsilon_{f}$. In addition, owing to the studied region being relatively small and the finite-adsorption restriction, the output concentration at steady state of all cases is the same with the input concentration.

For further studying the size effect of porous blocks on adsorption, a system with three types of porous blocks with varying areas is also investigated. It can be seen from the difference of Figures 5(a) and 5(b); without adsorption $\left(V_{m}=0\right)$, gas diffuses with the greatest rapidity if there is an optimum connectivity of nanopores. This was also confirmed by Figure 6, on the assumption that other factors being unchanged, the output concentration rate (Figure 6(a)), and the concentration change rate of porous blocks center (Figure 6(b)) decreases with increasing $V_{m}$. The rate of adsorption $R_{\mathrm{a}}$, as expected in (13), always increases with the difference between the saturation adsorption capacity $V_{m}$ and adsorbed amount $V$, leading to an increase in the total adsorbed amount. The adsorbed amount difference between each case with different $V_{\mathrm{m}}$ will become smaller over time, but it will last for quite a long time. Comparing the right column with the left column in Figure 5, as the size of porous blocks increases, so is the difficulty of gas fully distributed inside the media, suggesting that in the form of fast gas-solid adsorption, gas diffusion may consume more time inside porous media.

The Langmuir pressure effect on adsorption is shown in Figure 6(c). The Langmuir pressure varies directly with the adsorption rate constant, and with a larger Langmuir pressure, the adsorbed amount will drop in that the adsorption strength will become weaker with the increasing Langmuir pressure when the desorption rate constant is fixed, as expected from (14). Therefore, both the output

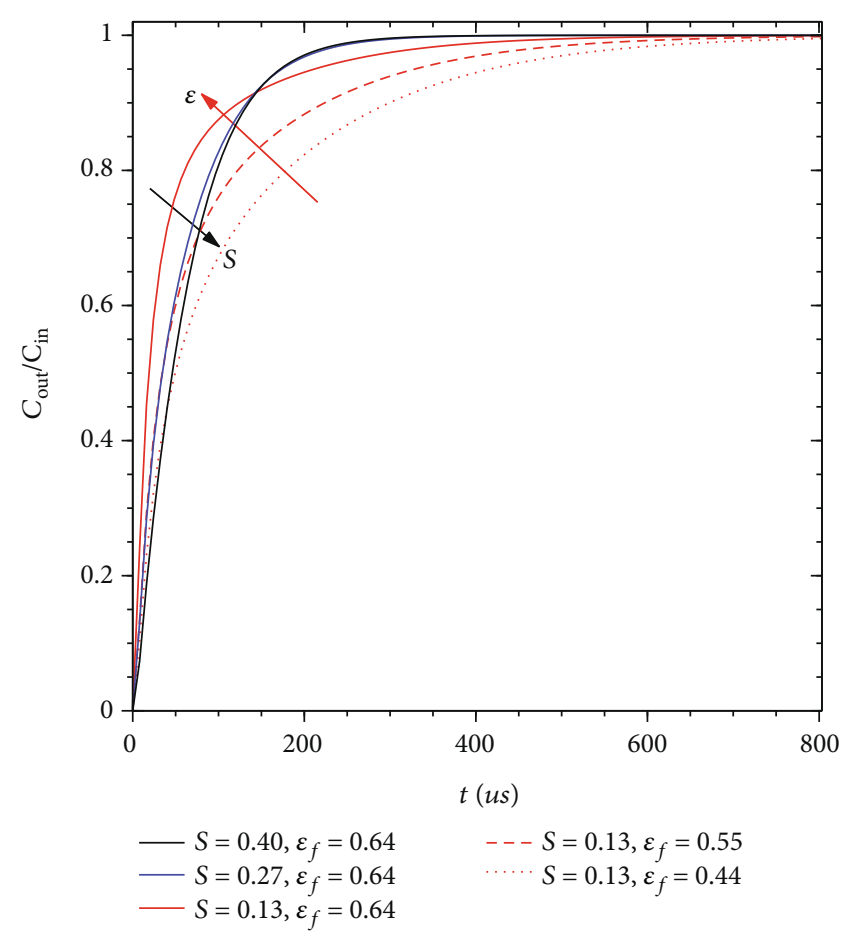

FIGURE 4: Results of the normalized output concentration for methane migration in porous media with adsorption under different porosities and specific surface areas, respectively. Note that the total output concentration $C_{\text {out }}$ is normalized by the input concentration $C_{\text {in }}$, and $C_{\text {in }}=C_{0} H$.

concentration and the central concentration increased with the Langmuir pressure.

4.3. Gas Migrations in the Reconstructed Coal Matrix. In order to gain a deeper insight into the gas transport behavior with adsorption in a coal gas reservoir, in this section, the methane gas flow in a reconstructed 2D structure was studied. Coal is a dual-porosity medium composed of matrix pores and fractures. Nanopores in the coal matrix are the source of coalbed methane, and the fracture between the matrices provides a channel for gas migration. Figure 7(a) shows the microstructures of the coal reproduced from the 

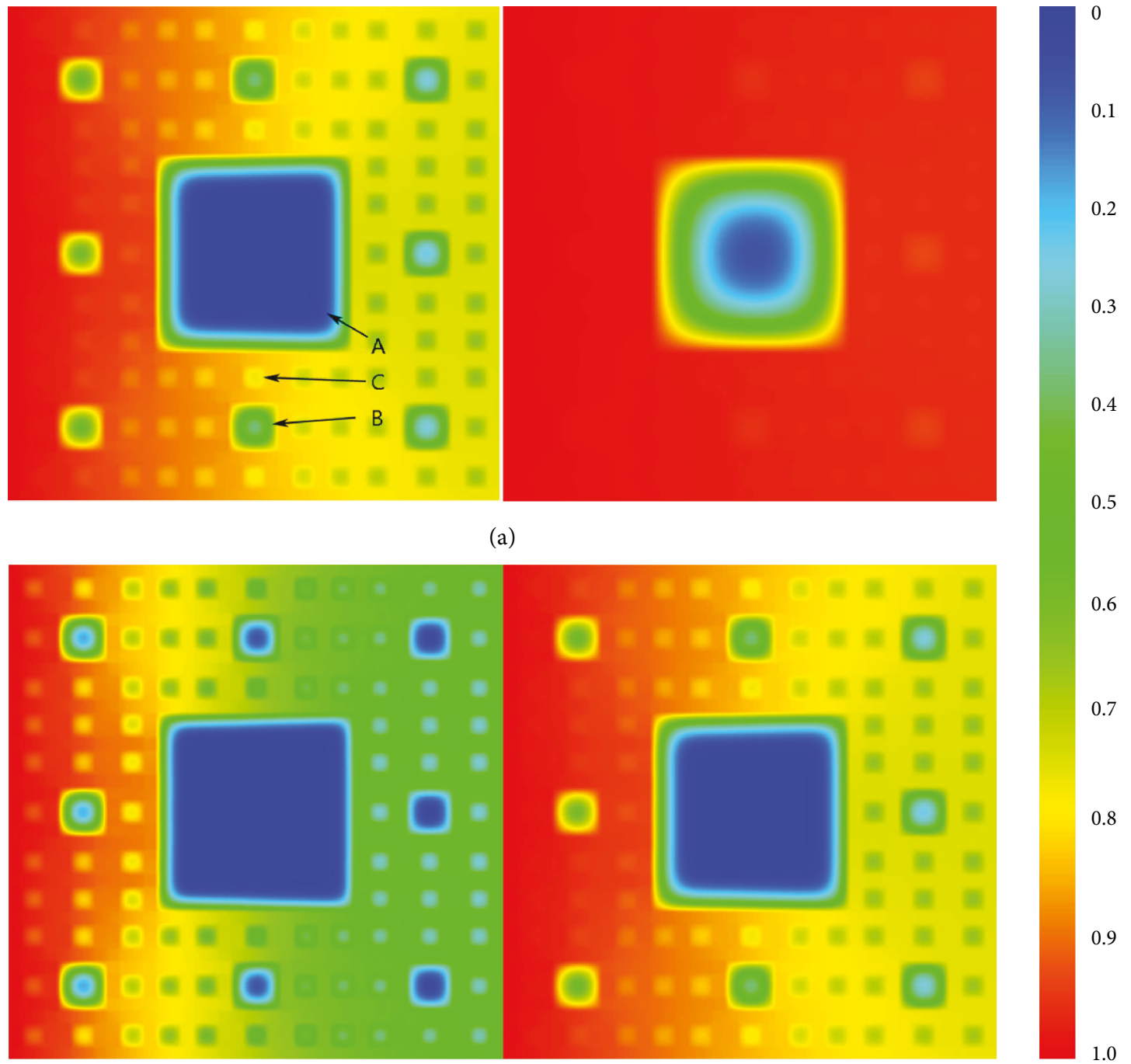

(b)

$\mathrm{m}^{3} / \mathrm{t}$

FIGURE 5: Concentration magnitude distribution in the porous media with and without adsorption effect. (a) No adsorption. (b) $p_{0}=10 \mathrm{MPa}$, $T=303.15 \mathrm{~K}, C_{\text {inlet }}=0.1 \mathrm{~mol} / \mathrm{m}^{3}$, and $V_{m}=30 \mathrm{~m}^{3} / \mathrm{t}, p_{L}=2 \mathrm{MPa}$. The matrix porosity of the solid matrix is 0.1 .

literature (reproduced from Figure 1 in [31]). It can be observed that numerous coal matrices with different sizes, shapes, and distributions have been divided by the fracture system. Both the size of the coal matrix and the fractures range from a few nanometers to several micrometers. In fact, countless nanopores were also distributed in the matrix. The distribution and geometry of the pores are associated with the coal rank, organic composition, and localized compaction, and thus, it is difficult to obtain a universal model from reconstruction. This paper reconstructed a porous medium shown in Figure 7(b) based on the distribution characteristics of the matrix-fracture system (as schematically shown in the red box of Figure $7(\mathrm{a})$ ), which roughly represents the microstructures of the coal composed of microfractures (white) and organic matrix (black). Besides, numerous nanosized pores of the organic matrix, which plays an important role in coal-methane adsorption, were also considered in the media. It can be found in (11) and (12) that the effective diffusion coefficient is proportional to both the porosity and the mean pore radius; thus, adjusting any one of them could achieve the different diffusivity of the porous media. Chen Jialiang and Yong [32] reported that the porosity of coal is related to the coal rank, and the variation range is 0.02 0.25. Besides, through coal samples from 25 areas in China, Fu and Wei [33] found that the mean pore radius spans from 3.8 to $15.2 \mathrm{~nm}$. In this section, the mean pore radius follows a unified value $(r=4 \mathrm{~nm})$, and the matrix porosities are chosen as $0.05,0.1,0.2$, and 0.4 . Note that the porosity of 0.4 may not be encountered in the coal samples, which is chosen here just for comparison. In the simulation, the boundary condition and input parameters are the same as those in Section 4.2 (Table 1).

Figure 8 shows the velocity magnitude distribution. When the porosity of the porous material is small $(\varepsilon=0.05$ on the left), the fluid flow along a strongly preferred pathway in the free flow region is due to the presence of the local flow 


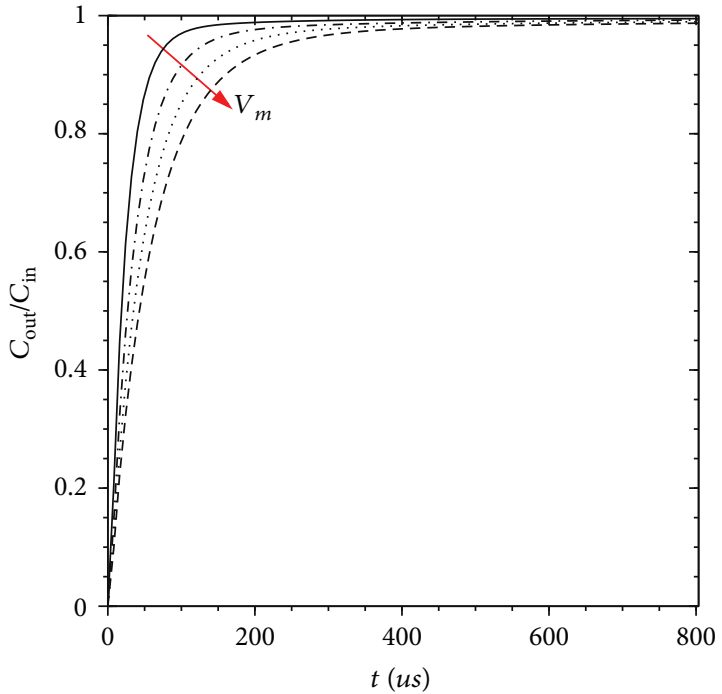

$\begin{array}{lll}\text { - Without adsorption } & \ldots . & V_{\mathrm{m}}=20 \mathrm{~m}^{3} / \mathrm{t} \\ .+. & V_{\mathrm{m}}=10 \mathrm{~m}^{3} / \mathrm{t} & \ldots--V_{\mathrm{m}}=30 \mathrm{~m}^{3} / \mathrm{t}\end{array}$

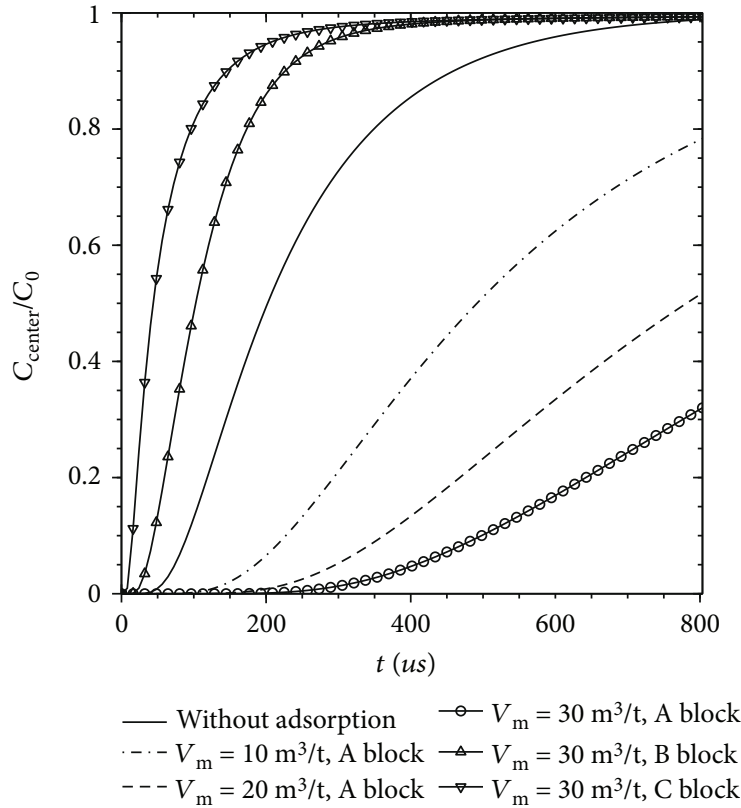

(b)

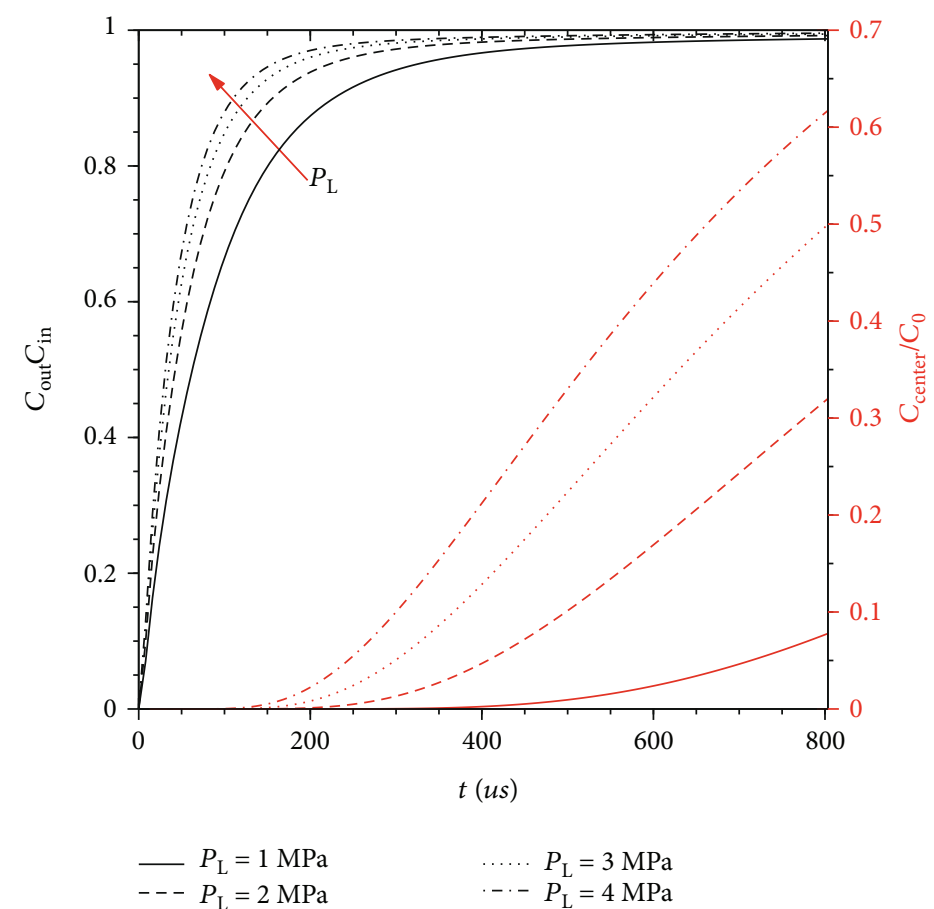

(c)

FIgURE 6: Concentration change over time. (a) Total concentration output at the outlet. (b) concentration through the center of blocks A, B, and C. (c) $p_{\mathrm{L}}$ effect; the red lines represent the corresponding central concentration of block A.

resistance. As porosity increases, the magnitude of the Darcy drag term in (3) will reduce, leading to a decrease in local resistance. Therefore, the fluid can pass through the porous media under this condition. When the porosity is relatively high $(\varepsilon=0.4$ on the right), the streamline almost appears along with a straight path. The results are consistent with the simulation results in [8], further suggesting the validity of our model.
Figure 9 summarizes the effect of the matrix porosity and the Langmuir pressure on the adsorbed amount magnitude distribution. The porosities of the porous solid for the set of images from the left to right columns are $0.05,0.1,0.2$, and 0.4 , respectively. The relatively higher porosity $(\varepsilon=0.4$ on the right column) makes the effective diffusivity of the organic matter stronger, increasing the rate of mass transfer. Therefore, the rate of gas diffusion in the porous material is 


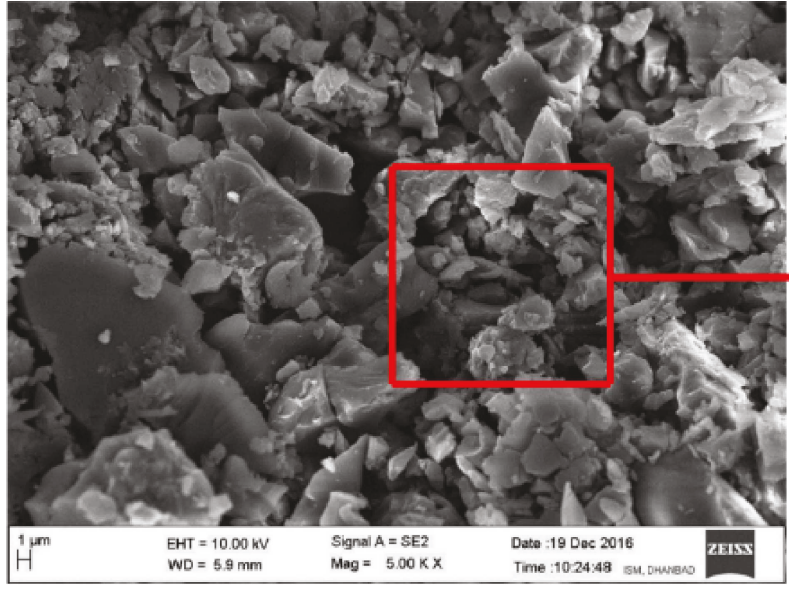

(a)

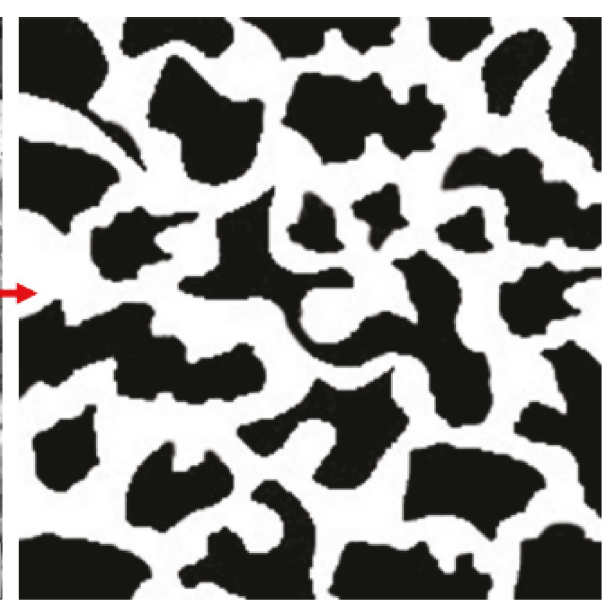

(b)

Figure 7: (a) The SEM image of a coal sample (reproduced from Figure 1 in [31]). (b) The reconstructed heterogeneous porous media with fractures (white) and organic matrix (dark) for simulation.

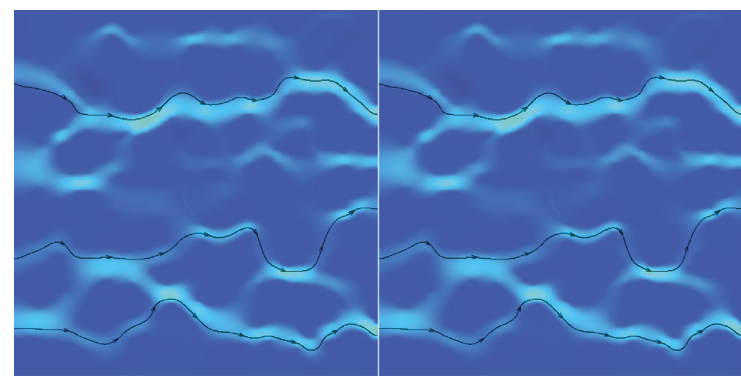

(a) (b)

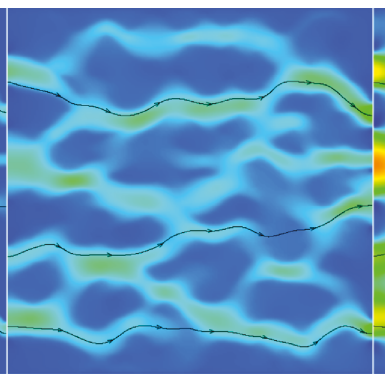

(c) $\times 10^{-5} \mathrm{~m} / \mathrm{s}$

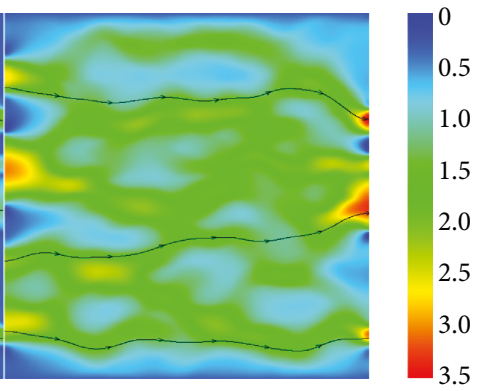

(d)

FIGURE 8: Velocity magnitude distribution in the porous media under different matrix porosities. (a) porosity $=0.05$, (b) porosity $=0.1$, (c) porosity $=0.2$, and $(\mathrm{d})$ porosity $=0.4$.

more significant and the dynamic adsorption equilibriumassociated region is more extensive.

The Langmuir pressure is in proportion to the ratio between desorption constant and adsorption constant, which is related to the adsorption strength of a porous matter. On the assumption that other parameters are kept constant, the Langmuir pressure determines the maximum adsorbed amount under dynamic equilibrium. In the simulation, we can vary the Langmuir pressure with a fixed desorption constant for studying the effect of the Langmuir pressure on the adsorbed amount, which means only the adsorption constant should be discussed. As can be seen on the right column of Figure 9, when the Langmuir pressure decreases, $k_{\mathrm{a}}$ becomes greater, which makes the adsorption strength greater. In consequence, the maximum adsorbed amount under dynamic equilibrium increases with the decreasing Langmuir pressure, as well as the total adsorbed amount, which also confirmed the results of Figure 6(c).

Moreover, medium resistance not only exists on fluid flow but also works for gas transport due to the diffusivity of the porous material far less than the free flow region. Thus, a diffusion peak curve will be formed based on the medium shape and distribution in the gas migrating processes, which might influence the rate of local gas-solid adsorption and also imply that the impact of the specific surface area and fracture porosity plays a key role on the adsorption process.

\section{Conclusion}

In this study, we proposed a coupled generalized NavierStokes equation and the advection-diffusion equation for gas migration in porous media which includes gas-solid adsorption and desorption. The effective Knudsen diffusion coefficient is introduced into the model for gas transport in the organic matrix of coal. The Langmuir adsorption rate equation is integrated into the source/sink term of the advection-diffusion equation for controlling the adsorption kinetic process of gas-solid adsorption and desorption. An LB model is developed for solving the coupled equations. 

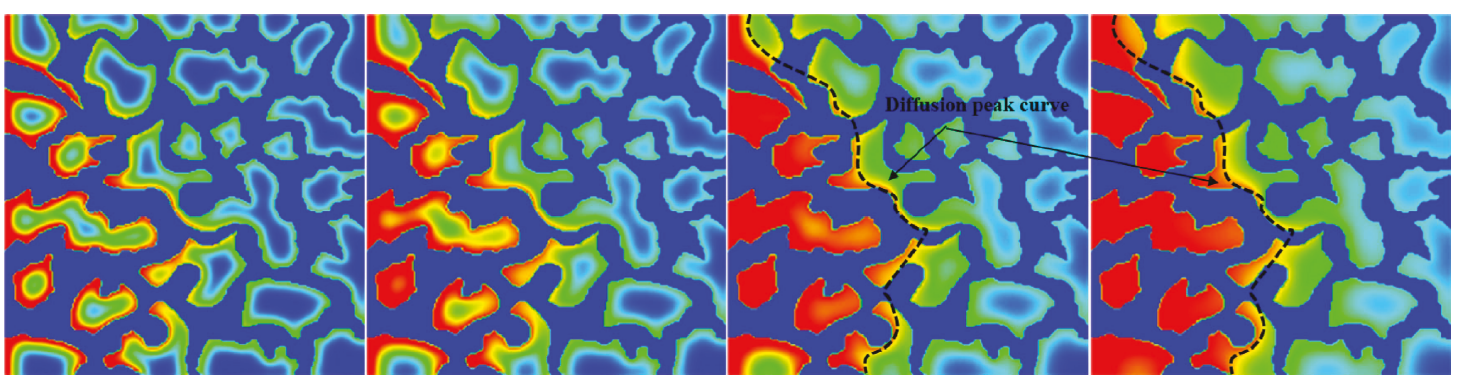

$\mathrm{m}^{3} / \mathrm{t}$

(a)
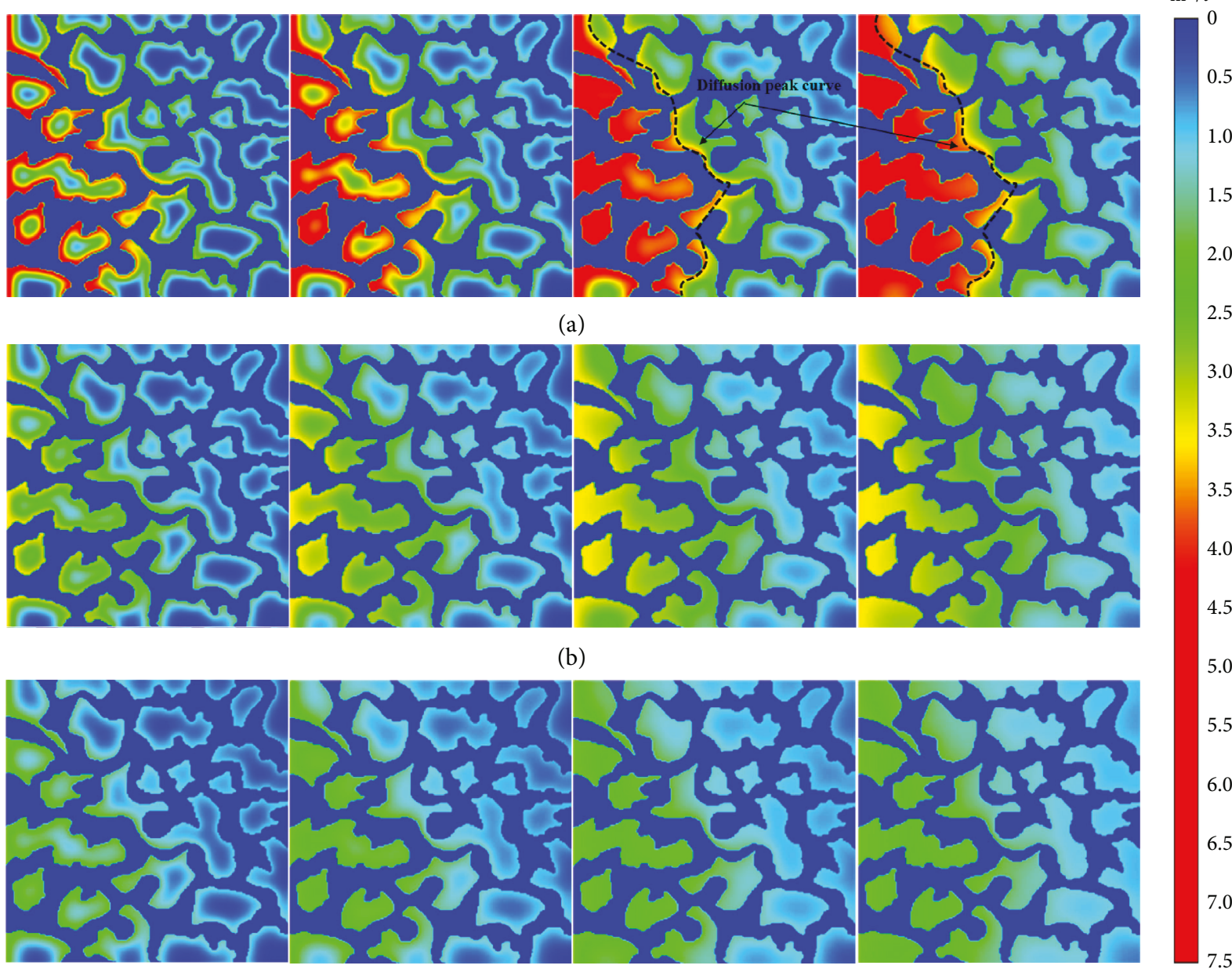

(b)
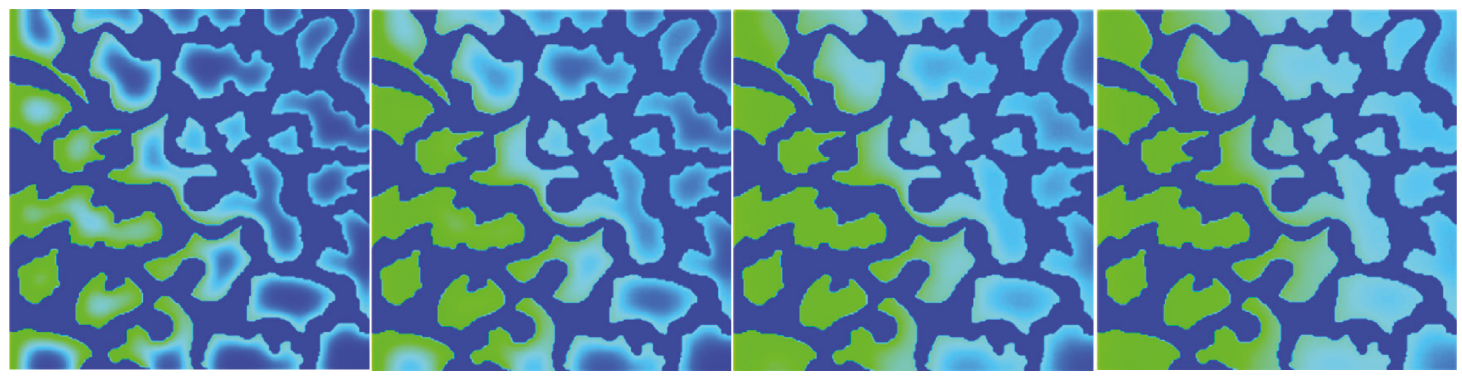

(c)

FIGURE 9: Magnitude and distribution of the adsorbed amount in the porous medium varying the matrix porosity and the Langmuir pressure, respectively. (a) $p_{\mathrm{L}}=1 \mathrm{MPa}$, (b) $p_{\mathrm{L}}=2 \mathrm{MPa}$, and (c) $p_{\mathrm{L}}=3 \mathrm{MPa}$. According to the isothermal adsorption equation in (14), the maximum adsorbed amounts in cases (a), (b), and (c) are 7.6, 4.4, and $3.1 \mathrm{~m}^{3} / \mathrm{t}$, respectively. The porosities of the permeable solid from the left to right columns are $0.05,0.1,0.2$, and 0.4 , respectively.

Simulations of the array-distributed regular solid blocks with different specific surface area, fracture porosity, matrix size, and saturation adsorption capacity are investigated. The results show that the adsorption effect becomes stronger as the specific surface area and the fracture porosity increase or the matrix size decreases. Moreover, the effect of saturation adsorption amount shows an opposite trend as the fracture porosity due to adsorption rate increases with the difference between saturation adsorption capacity and the adsorbed amount, and the impact of Langmuir pressure shows a similar trend as the fracture porosity. Simulation results of the reconstructed coal matrix confirm that methane-coal adsorption is influenced by the matrix porosity/permeability and the Langmuir pressure, as well as the geometric complexity. A higher matrix porosity/permeability or lower Langmuir pressure increases the adsorption rate and the corresponding affecting area. The appearance of the diffusion peak curve indicates that geometric complexity, including matrix size, shape, and distribution, may play a significant role in the adsorption-desorption process.
Our model can be applied to a wide range of simulation in unconventional natural gas exploitation. However, future work is still needed including more realistic effects in coal such as gas desorption and matrix deformation.

\section{Data Availability}

The data used to support the findings of this study are included within the article.

\section{Additional Points}

Highlights. (1) A double-distribution Lattice Boltzmann model for solving the coupled generalized Navier-Stokes equation and advection-diffusion equation with respect to the gas-solid dynamic adsorption process is proposed for multicomponent gas migration in the unconventional reservoir. (2) The effective diffusion coefficient is introduced to the model of gas transport in the porous media. The Langmuir adsorption rate equation is employed to control 
the adsorption kinetic process of gas-solid adsorption. (3) The effect of porosity/permeability, the saturation adsorption amount, the Langmuir pressure, and the geometric complexity of methane-coal adsorption dynamic processes are numerically investigated.

\section{Conflicts of Interest}

The authors declare that they have no conflicts of interest.

\section{Acknowledgments}

This work was supported by the National Natural Science Foundation of China (Grant no. 41472130) and the National Key Research and Development Program of China (Grant no. 2016YFC0600704).

\section{Supplementary Materials}

(1) This program is a $2 \mathrm{D}$ generalized lattice Boltzmann model code using a double-distribution function to simulate gas transport in a coal reservoir. (2) The whole project is composed of 1.par and GLBM.f90. 3. "1.par" is a header file, where some common blocks are defined; all the subroutines are integrated into "GLBM.f90," where "subroutine FlowField" is employed to reconstruct the images of $2 \mathrm{D}$ porous media after binarization by MATLAB, "subroutine evolution" and "subroutine diffusion" are implemented to govern the fluid bulk flow and diffusion, respectively, and "subroutine initial" and "subroutine output" are used to initialize the flow field and record the simulation results, respectively. (4) Before running this project, please create a new subfolder "out" under the working directory. (5) In the simulation, all the parameters are dimensionless, and the output data can be dimensionalized using the principle of similitude. (Supplementary Materials)

\section{References}

[1] D. Ritter, D. Vinson, E. Barnhart et al., "Enhanced microbial coalbed methane generation: a review of research, commercial activity, and remaining challenges," International Journal of Coal Geology, vol. 146, pp. 28-41, 2015.

[2] T. A. Moore, "Coalbed methane: a review," International Journal of Coal Geology, vol. 101, pp. 36-81, 2012.

[3] K. S. W. Sing, "Reporting physisorption data for gas/solid systems with special reference to the determination of surface area and porosity (Recommendations 1984)," Pure and Applied Chemistry, vol. 57, no. 4, pp. 603-619, 1985.

[4] B. Hodot, Outburst of Coal and Coalbed Gas (Chinese Translation), China Coal Industry Press, Beijing, 1966.

[5] R. M. Roque-Malherbe, Adsorption and Diffusion in Nanoporous Materials, CRC press, 2007.

[6] J. E. Warren and P. J. Root, "The behavior of naturally fractured reservoirs," Society of Petroleum Engineers Journal, vol. 3, no. 3, pp. 245-255, 1963.

[7] Y. Cai, D. Liu, Z. Pan, Y. Yao, J. Li, and Y. Qiu, "Pore structure and its impact on $\mathrm{CH} 4$ adsorption capacity and flow capability of bituminous and subbituminous coals from Northeast China," Fuel, vol. 103, pp. 258-268, 2013.
[8] L. Chen, W. Fang, Q. Kang, J. de'Haven Hyman, H. S. Viswanathan, and W. Q. Tao, "Generalized lattice Boltzmann model for flow through tight porous media with Klinkenberg's effect," Physical Review E, Statistical, Nonlinear, and Soft Matter Physics, vol. 91, no. 3, article 033004, 2015.

[9] Z. Guo and T. S. Zhao, "Lattice Boltzmann model for incompressible flows through porous media," Physical Review E, Statistical, Nonlinear, and Soft Matter Physics, vol. 66, no. 3, article 036304, 2002.

[10] Z. Tian, H. Xing, Y. Tan, S. Gu, and S. D. Golding, "Reactive transport LBM model for $\mathrm{CO}_{2}$ injection in fractured reservoirs," Computers \& Geosciences, vol. 86, pp. 15-22, 2016.

[11] J. Wang, L. Chen, Q. Kang, and S. S. Rahman, “Apparent permeability prediction of organic shale with generalized lattice Boltzmann model considering surface diffusion effect," Fuel, vol. 181, pp. 478-490, 2016.

[12] Y. Ning, S. He, H. Liu, H. Wang, and G. Qin, "Permeability prediction considering surface diffusion for gas shales by lattice Boltzmann simulations on multi-scale reconstructed digital rocks," in International Petroleum Technology Conference, Bangkok, Thailand, November 2016.

[13] G. Falcucci, S. Melchionna, S. Ubertini, and S. Succi, "Complex flow simulation via lattice Boltzmann method," in Handbook of Fluid Dynamics, CRC Press, 2016.

[14] Y. Gan, A. Xu, G. Zhang, and S. Succi, "Discrete Boltzmann modeling of multiphase flows: hydrodynamic and thermodynamic non-equilibrium effects," Soft Matter, vol. 11, no. 26, pp. 5336-5345, 2015.

[15] S. Succi, "Lattice Boltzmann beyond Navier-Stokes: where do we stand?," AIP Conference Proceedings, A. Ketsdever and H. Struchtrup, Eds., AIP Publishing, 2016.

[16] P. Nithiarasu, K. N. Seetharamu, and T. Sundararajan, "Natural convective heat transfer in a fluid saturated variable porosity medium," International Journal of Heat and Mass Transfer, vol. 40, no. 16, pp. 3955-3967, 1997.

[17] Q. Liu and Y.-L. He, "Lattice Boltzmann simulations of convection heat transfer in porous media," Physica A: Statistical Mechanics and its Applications, vol. 465, pp. 742-753, 2017.

[18] E. Fathi and I. Y. Akkutlu, "Lattice Boltzmann method for simulation of shale gas transport in kerogen," SPE Journal, vol. 18, no. 01, pp. 27-37, 2013.

[19] X. He, N. Li, and B. Goldstein, "Lattice Boltzmann simulation of diffusion-convection systems with surface chemical reaction," Molecular Simulation, vol. 25, no. 3-4, pp. 145-156, 2000.

[20] M. Pillalamarry, S. Harpalani, and S. Liu, "Gas diffusion behavior of coal and its impact on production from coalbed methane reservoirs," International Journal of Coal Geology, vol. 86, no. 4, pp. 342-348, 2011.

[21] Z. Yang, W. Wang, M. Dong et al., "A model of dynamic adsorption-diffusion for modeling gas transport and storage in shale," Fuel, vol. 173, pp. 115-128, 2016.

[22] X. Zhang, Z. Du, and P. Li, "Physical characteristics of highrank coal reservoirs in different coal-body structures and the mechanism of coalbed methane production," Science China Earth Sciences, vol. 60, no. 2, pp. 246-255, 2017.

[23] F. Javadpour, "Nanopores and apparent permeability of gas flow in mudrocks (shales and siltstone)," Journal of Canadian Petroleum Technology, vol. 48, no. 08, pp. 16-21, 2009.

[24] S. M. Kang, E. Fathi, R. J. Ambrose, I. Y. Akkutlu, and R. F. Sigal, "Carbon dioxide storage capacity of organic-rich shales," SPE Journal, vol. 16, no. 04, pp. 842-855, 2011. 
[25] D. D. Do and K. Wang, "Dual diffusion and finite mass exchange model for adsorption kinetics in activated carbon," AICHE Journal, vol. 44, no. 1, pp. 68-82, 1998.

[26] D. D. Do and K. Wang, "A new model for the description of adsorption kinetics in heterogeneous activated carbon," Carbon, vol. 36, no. 10, pp. 1539-1554, 1998.

[27] J. Wang, Q. Kang, Y. Wang, R. Pawar, and S. S. Rahman, “Simulation of gas flow in micro-porous media with the regularized lattice Boltzmann method," Fuel, vol. 205, pp. 232-246, 2017.

[28] X. Su, X. Lin, S. Liu, M. Zhao, and Y. Song, "Geology of coalbed methane reservoirs in the Southeast Qinshui Basin of China," International Journal of Coal Geology, vol. 62, no. 4, pp. 197210, 2005.

[29] C. D. Bohn, S. A. Scott, J. S. Dennis, and C. R. Müller, "Validation of a lattice Boltzmann model for gas-solid reactions with experiments," Journal of Computational Physics, vol. 231, no. 16, pp. 5334-5350, 2012.

[30] L. Zhou, Z. G. Qu, L. Chen, and W. Q. Tao, "Lattice Boltzmann simulation of gas-solid adsorption processes at pore scale level," Journal of Computational Physics, vol. 300, pp. 800813, 2015.

[31] M. Asif, N. Paul, D. C. Panigrahi, and K. Ojha, "Pore characterization of coal of Jharia coalfield for coalbed methane using pore image analysis," in 79th EAGE Conference and Exhibition 2017, Paris, France, June 2017.

[32] S. Z. Chen Jialiang and Q. Yong, Energy Geology, China University of Mining and Technology press, 2004.

[33] Y. Q. X. H. Fu and C. T. Wei, Coalbed Gas Geology, China University of Mining and Technology Press, 2007. 

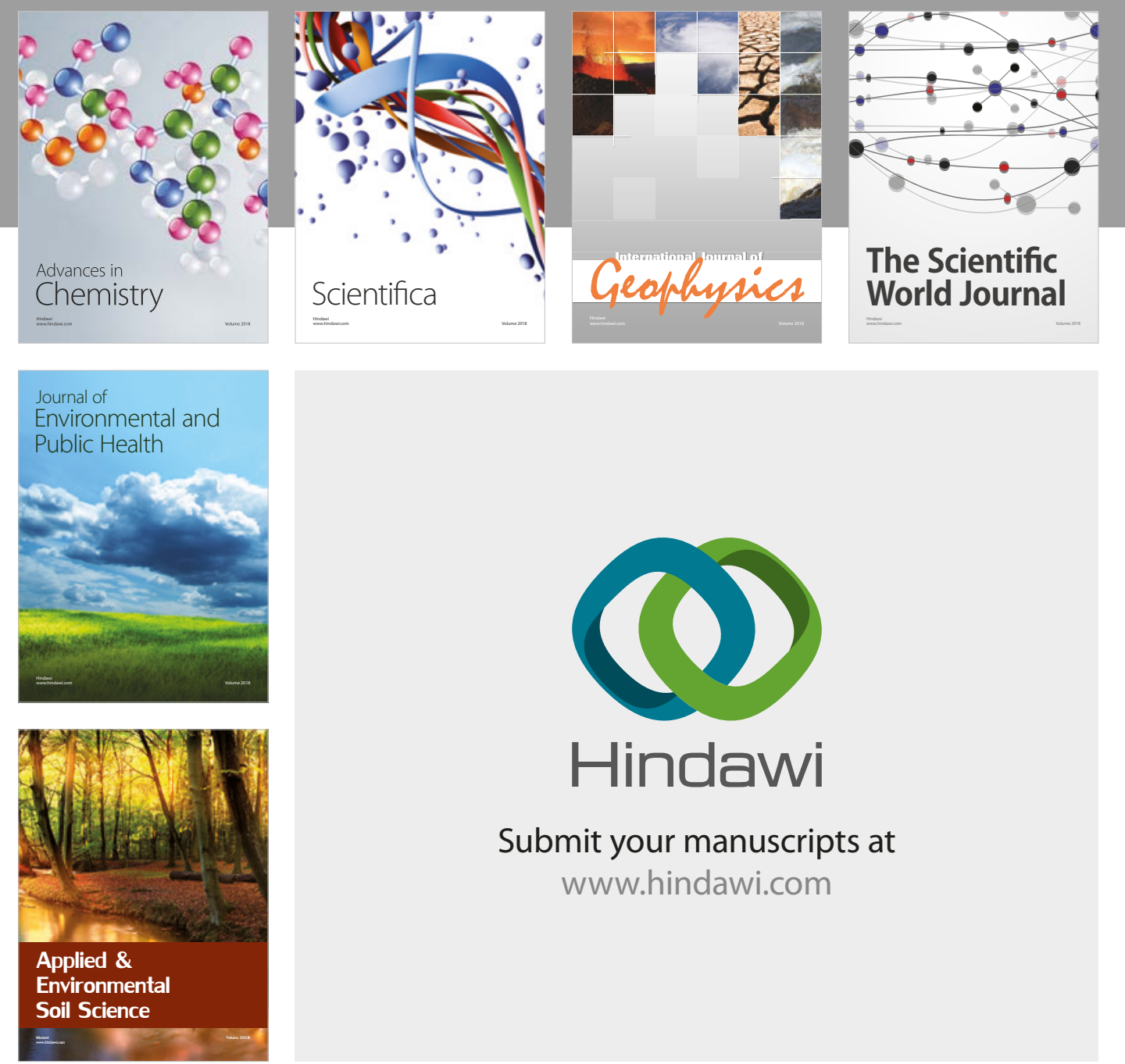

The Scientific

\section{World Journal}
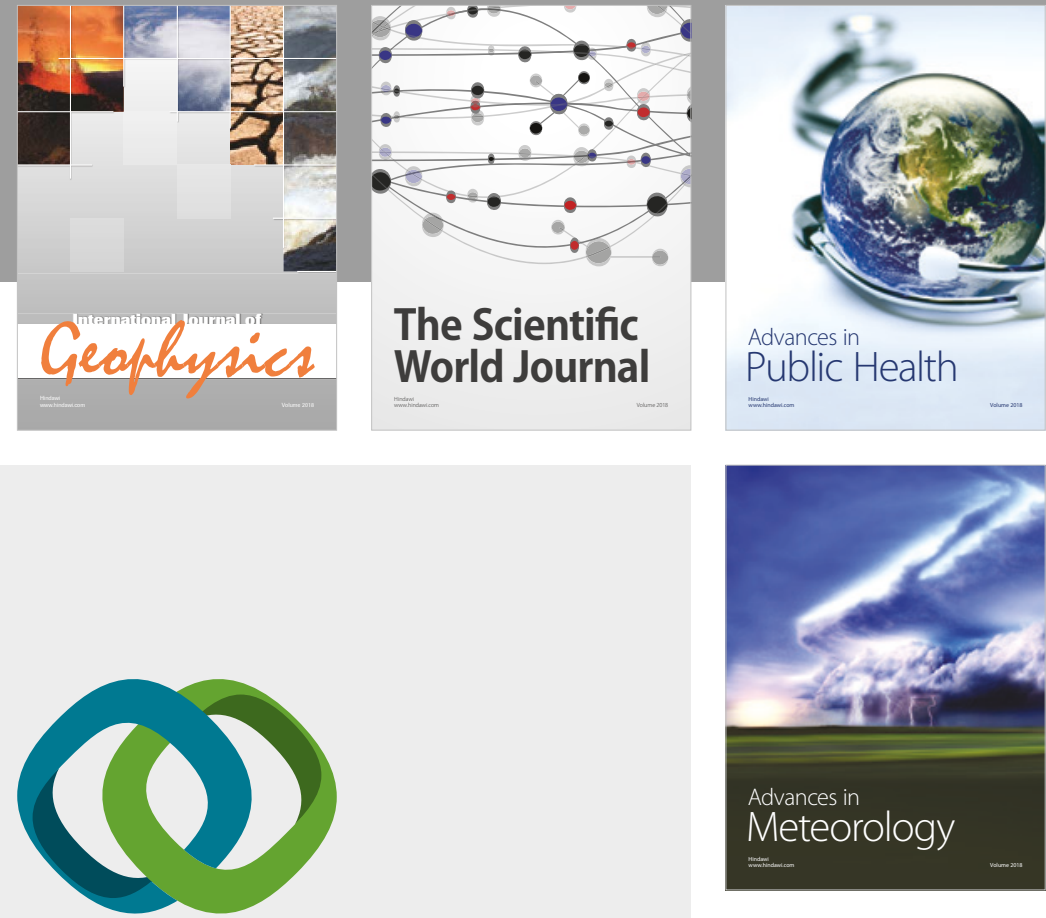

Advan

Public Health

\section{Hindawi}

Submit your manuscripts at

www.hindawi.com
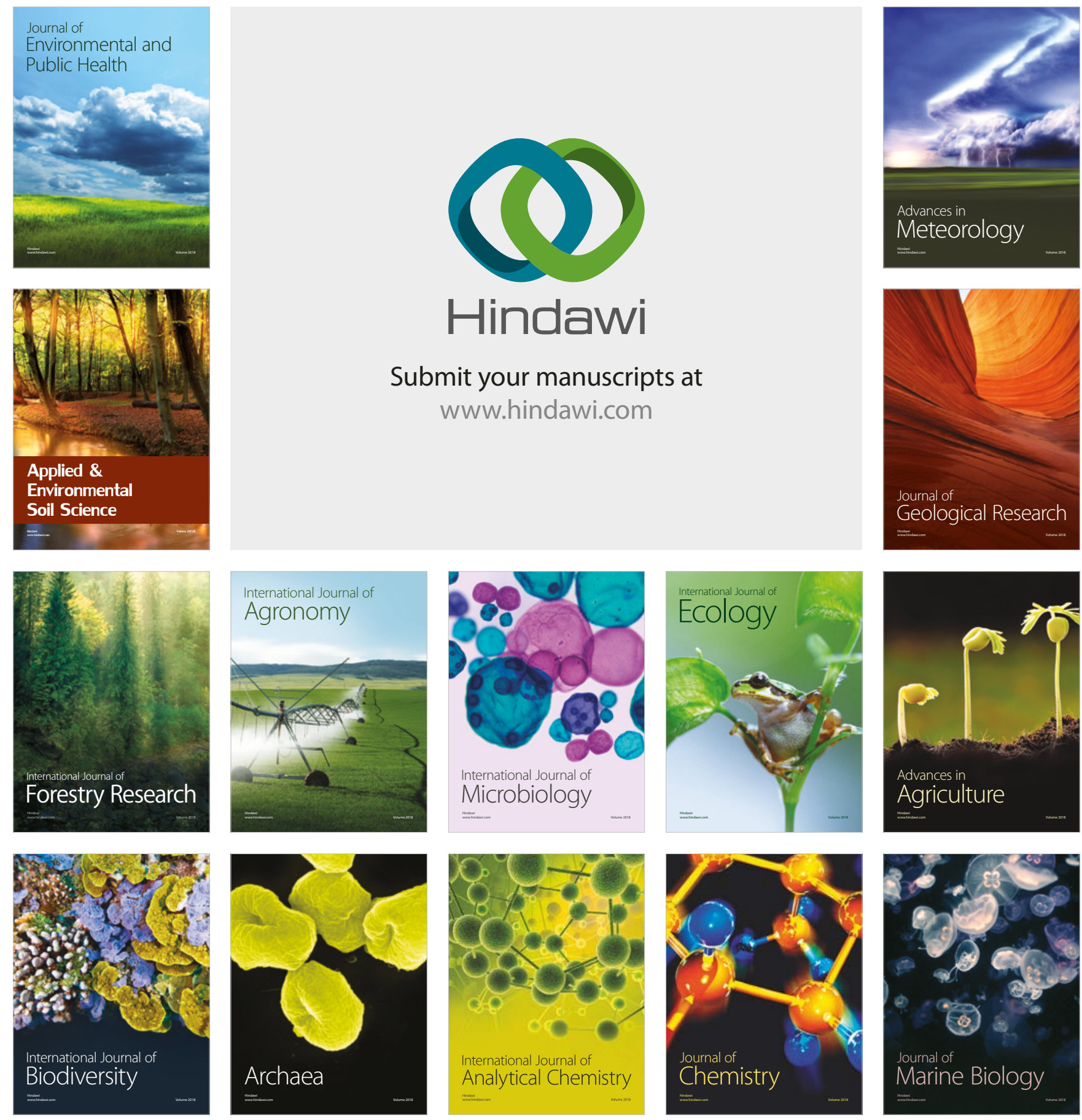